\title{
Drum Circles and the Effect on Well-being in a Community Setting
}

\author{
Ashton Mason \\ College of the Arts
}

Faculty Mentors: Jennifer Lee, and Jill Sonke, Center for Arts in Medicine

\begin{abstract}
Well-being plays an essential role in a healthy community. Participatory arts interventions, like drum circles, act as a popular activity to address well-being. These activities lead to social resilience, emotional completeness, and positive mental health benefits. The literature in the arts in health demonstrates the specific ways a drum circle may affect well-being through socialization, rhythmic entrainment, and expression. Although there are great resources, many lacks the details to facilitate a drum circle as it pertains to specific techniques and styles often utilized in percussion in the field of arts in health. To explore the topic of well-being and drum circles, a mixed-methods research design was chosen. This project uses a convergent mixed method design to collect quantitative data from the Warwick Edinburgh Mental Well-being Scale and the qualitative data from a focus group. The results are compared with each other with the hopes of yielding similar themes from each data set. The Warwick Edinburgh Mental Wellbeing scale results demonstrate an increase of the average well-being score after the four-week intervention by 2.88 points. The focus group's final themes were 1) the core of the drum circle is driven from the facilitator fostering a strong sense of community that supports being inclusionary, respectful, and social; 2) Learning new skills in a community group benefits participants confidence because a) the curriculum progresses naturally and easily and $b$ ) rhythm is an innate and natural part of everyday life for individuals making it easier for individuals to feel comfortable creating rhythms.

Keywords: Well-being; Drumming; Percussion; Community Art Engagements; Drum Interventions; Drum Circles; Health
\end{abstract}

\section{Introduction}

Well-being is at the forefront of innovative health initiatives as researchers explore the benefits and best practices of increasing well-being in community health and healthcare settings (Langevin, 2019). Well-being is defined as "concerned with the balance between positive affect and negative affect, along with perceived satisfaction with one's life" (Ascenso et al., 2018). The field of arts in health utilizes the arts to enhance the well-being and health across these settings (Lambert, Betts, Rollins, Sonke \& Swanson, 2017). This report explores active drum circles and its impact on well-being while furthering the discussion of best practices. 
Well-being is a positive state of mind and music has a role to play in developing this positive interaction (Thompson, 2015, p. 175.). Thompson (2015) lists ten reasons why music benefits well-being. Music is: ubiquitous, emotional, engaging, distracting, physical, ambiguous, social, communicative, manipulative and personal (Thompson, 2015, p. 169-236.). As it pertains to drumming specifically, rhythmic entrainment models demonstrate the attentional synchrony of rhythmic events, which ultimately drives the neural oscillations in one's brain (Thompson, 2015). Neural oscillation relates to individual connection with rhythmic speech and, ultimately, drum circles' pull (Thompson, 2019, p. 187).

Recreational art engagements, like drum circles, are well received across communities especially with the concept of healing holistically through following a cultural path, regaining a balanced life, and sharing this experience with others in your community (Hunter et al., 2006; Davies, Knuiman, \& Rosenberg, 2016). Data suggests that community members who partake in these engagements, whether the participants are passively listening or actively playing, have a higher sense of well-being than individuals who did not (Davies et al., 2016). With evaluation on mental well-being, depression, anxiety, and social resilience, participants in drum circles demonstrate a decrease in depression and anxiety as well as an increase in social resilience and mental well-being (Fancourt et al., 2016).

The data presented demonstrates the positive effects of drum circles, though much of the research lacks specificity in methodology and facilitation. Engrossing the participants in culture, style, and specific instruction are explored, yet not detailed (Perkins et al., 2016; Davies et al., 2016; Mungas \& Silverman, 2014; Fancourt et al., 2016). Phrases like "non-specified drumming patterns" are often utilized which give a facilitator no direction in styles or specific rhythms (Perkins et al., 2016).

This report strives to fill the gap in the literature supporting the pre-existing evidence that demonstrates drum circles impact on well-being and furthers the field by producing a thorough curriculum (Davies et al., 2016; Faulkner, 2017; Mungas \& Silberman, 2014; Fancourt et al., 2016). 


\section{Materials and Methods}

\section{Consent}

An institutional Review Board (IRB) consent was submitted and accepted at the University of Florida at the start of the project. The goal of the IRB is to protect the rights and welfare of the participants in human subject studies like this one ("IRB Home", 2020). A consent form was given to all participants at the beginning of the study and signed individually.

\section{Participants}

The study population was at most seven adults who live in the Gainesville community areas. All participants were eighteen years or older. Age was the only restriction with the hopes of creating an inclusive and diverse population. A low population was chosen for the safety of the participants because the Center for Disease Control and Prevention (2020) recommends smaller gatherings and social distancing due to the COVID-19 virus.

\section{Study Design}

This project will be using a mixed methods research design (Creswell \& Creswell, 2018). This approach gathers multiple data sources and combines them to gain more insight into the questions the project is attempting to answer: How and why we can create an effective drum circle that will impact well-being (Creswell \& Creswell, 2018)? A convergent mixed methods design collected the quantitative and qualitative data and compared them with the hopes of yielding similar themes between each data set to validate the results (Creswell \& Creswell, 2018).

The quantitative data will be found using the Warwick Edinburgh Mental Well-being Scale (WEMEBS), and the qualitative data will be gathered from a focus group ("WEMWBS: 14 item vs 7-item scale”, 2019; Schoonenboom \& Johnson, 2017). The WEMWBS has been validated through psychometric tests like construct validity, confirmatory factor analysis, and test-rests reliability although, a limitation is that a gold standard measure does not exist as of right now. Many researchers have continued to utilize the WEMWBS when evaluating music's impact on wellbeing despite this limitation. The first WEMWBs will be given at the first intervention in paper form ("WEMWBS: 14-item vs 7-item scale", 2019). The same procedure was followed in the last intervention for the purpose of providing consistency ("WEMWBS: 14-item vs. 7-item scale", 2019). Data from both scales were analyzed to evaluate how the participants' mental well-being changed over time (“Conceptual Framework”, 2019). The WEMWBS is a 14-item 
scale that created the full picture of improvement or deterioration where the results are evaluated through a point system ("WEMWBS: 14-item vs 7-item scale", 2019). The scale covers eudaimonia and hedonic well-being, subjective well-being, and psychological functioning (“Conceptual Framework”, 2019).

The focus group will be held after the final drum circle. The questions will be pre-determined based on the core themes of the project: well-being, drum circles, specific techniques, specific styles learned, and the activities used (Onwuegbuzie et al., 2009). The transcription of the focus group was done immediately following for the purpose of consistency in understanding the recording (Onwuegbuzie et al., 2009). An inductive coding approach where data is analyzed through a process where the research interprets the data to develop consistent concepts and themes (Onwuegbuzie et al., 2009). This process evolved through open coding where relevant words and phrases associated with the research topic will be explored (Onwuegbuzie et al., 2009). Within the first cycle, labels, words, and phrases were noted in a line-by-line process. A codebook was developed and condensed within the second cycle (Onwuegbuzie et al., 2009). The third cycles utilized axial coding where sub-categories and themes emerged (Onwuegbuzie et al., 2009). Once there were identifiable themes, a narrative was created by the researcher who conducted the coding in triangulation with the quantitative data found from the WEMWBS (Creswell, \& Creswell, 2018).

\section{Materials Purchased}

Instrumentation has been specified to the Remo-Versa drum series which is a recommended brand for drum circles by Simon Faulkner (Faulkner, 2017). This drum series includes djembes, timbaus, and tubanos which are larger bodied drums that allow for deeper sounds and is more appropriate for group playing because the sound will be louder compared to high-toned drums and it can be more closely related to natural sounds like heart beats (Faulkner, 2017). Lollipop drumsticks, a great tool for individuals who felt uncomfortable playing the drums with their bare hands, were purchased. In coordination with the "Health Requirements" (2021) at the University of Florida, face coverings, sanitization wipes, and hand sanitizer were also purchased for the participants' use. The University of Florida Center for Arts in medicine provided the chairs for the drum circle. Small hand-held percussion instruments like egg shakers, frame drums, maracas, tambourines, and castaets, were also borrowed from the Center. 


\section{Curriculum}

Lesson 1:

1. Check in

a. An introduction of the consent form and the project details will be stated to the group.

b. Stretching with an audio soundtrack to get the body ready for movement will be led by the facilitator.

c. Name Game

i. Go around the circle and create a rhythm to associate with your name. Once the name is said, the group repeats the name and their rhythm back to the participant with the goal of positive socialization.

1. Example

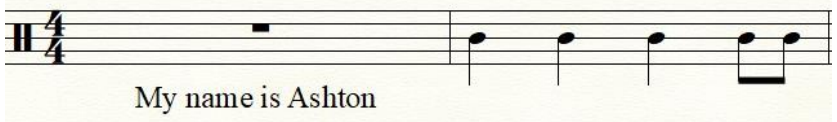

Figure 1. Name Game Example 1

d. I like

i. Go around the circle and create a rhythm to associate with something you like (food, movies, games, etc.). The group repeats what the participant likes and the rhythm.

1. Example

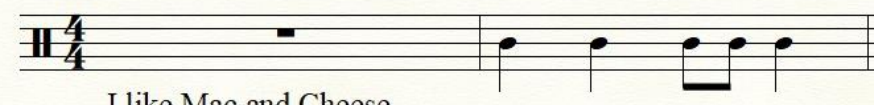

I like Mac and Cheese

Figure 2. Name Game example 2.

2. Identify and demonstrate good hand drumming technique

a. What instruments do we have?

i. Timbau

ii. Tubano 


\section{iii. Djembe}

1. The drums are made from weather resistant, durable, and stable Acousticon which is a type of material. They are manufactured by Remo.

b. How to hit the drum

i. With your hands or mallets on the head of the drum.

c. Sounds

i. Tone

1. These are medium pitched. To achieve this sound, one must hit the edge of the drum slightly more to the center. Keep the fingers flat and move the whole arm onto the drum rather than just using your wrist.

ii. Bass

1. This is the lowest pitch. Hit the center of the drum with the palm of your hand.

iii. Mute

1. Strike the drum and do not lift your palm off the head of the drum.

\section{Simon Says}

a. One person is the leader and calls out the sounds. Everyone else must follow the leader and play the sounds, but only when Simon says.

i. For Example: Simon says bass tone tone bass.

\section{Solo/ Improvisation Activity}

a. The whole group plays a basic rhythm. One-by-one participants solo over the group's base rhythm. It is important to explain that the purpose of this is to explore the musical space rather than playing perfectly in time or with perfect technique.

i. Examples of base rhythms

1.

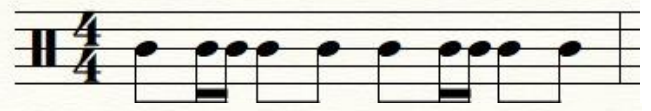

2.

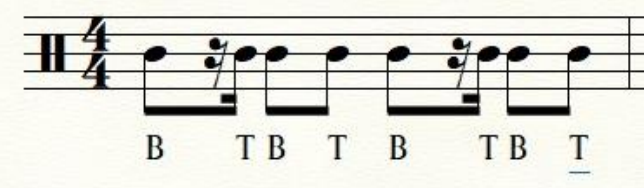

Figure 4. Base Rhythm Example 2. 
3.



Figure 5. Base Rhythm Example 3.

4.

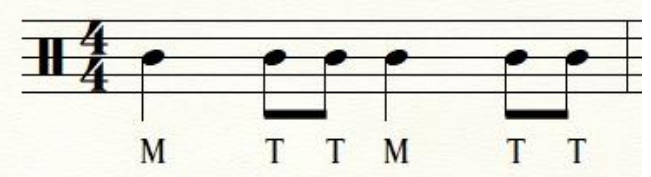

Figure 6. Base Rhtyhm Example 4.

5. Close Out

a. Relaxation rhythms/ sounds

i. Play along to an orchestrated song where participants can explore their own sound space which is the space in which they create sound through their own creative energy without following specific guidelines.

b. Summary/ Check in

Lesson 2:

1. Check in

a. Stretch with soundtrack

b. Name Game

2. Recap of techniques/ sounds

3. Simon Says

4. Solo/ Improvisation Activity

5. Afro Cuban Rumba

a. What is it?

i. This is originally a ballroom dance of Afro-Cuban fold dance origin (Daniel, 1991).

ii. Originating in the late $19^{\text {th }}$ century among the black population of the eastern Cuban Province of Oriente, the style includes vocals, instrumentals, 
and dance derived from African and Spanish influences. The Afro-Cuban rumba developed in the black urban slums of Cuba. (Daniel, 1991).

1. This style was chosen for its popular rhythmic model making it a familiar style to the participants. Familiarity with the sound makes the participants more comfortable to play along with.

iii. Rhythm

1.
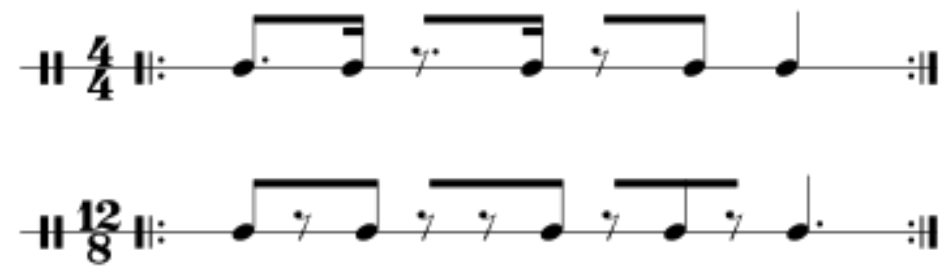

Figure 7. Afro-Cuban Rhythm Example.

iv. Improvisation game centered around this rhythm

1. Make sure participants are playing without being rigid by demonstrating how to keep the body loose with free movement. Also, when teaching this rhtyhm, do not teach the participants about time signatures, rather teach the rhythm by sound, and feel.

6. Free Style over Audio Recording

a. Allow the participants to choose which song they would like to play along with.

7. Closing

a. Relaxation Exercise

b. Check out

Lesson 3

Objectives:

1. Check-in

a. Stretch with soundtrack

2. Recap of techniques

a. Introduce Flam

i. Flam consists of two single strokes played by alternating hands. The first hit is a quieter note followed by a louder primary hit with the other hand almost simultaneously. 
3. Recap of African Rumba style

a. Solo above the base rhythm like in Lesson 2 .

4. Learn the style of the Gahu

a. Gahu is a recreational style of music of the people of Ewe of Ghana, Togo and Benin (“Traditional Drumming \& Dance from Ghana: Gahu”, 2019). There are many renditions and interpretations throughout the neighboring villages (“Traditional Drumming \& Dance from Ghana: Gahu”, 2019).

b. A traditional gahu drumming ensemble is comprised of six different instrument types, each with a distinct construction, sound, and rhythmic character (“Traditional Drumming \& Dance from Ghana: Gahu”, 2019).

c.

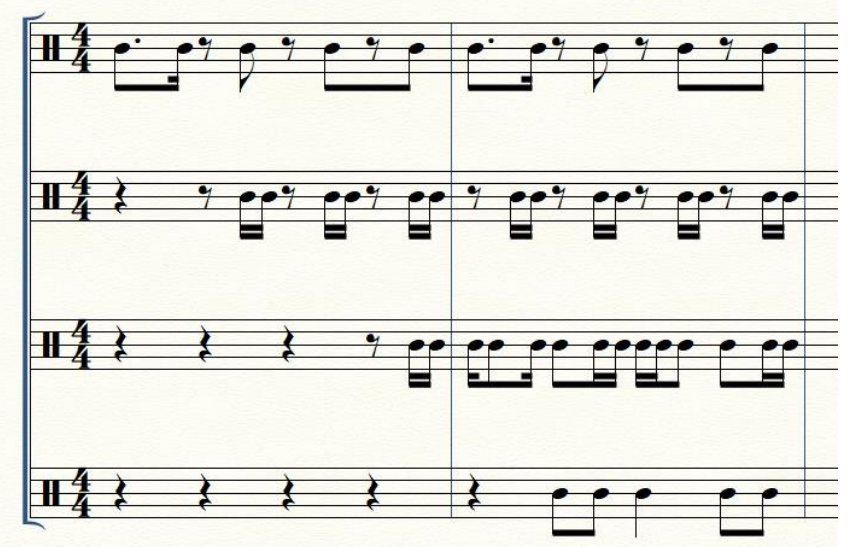

Figure 8. Example of Gahu Rhythms overlapped with each other.

i. The purpose of learning this style is to build upon sound textures while representing a non-westernized style. Having a culturally diverse curriculum is beneficial because it allows the participants to gain a better understanding of others when working in a social situation.

1. Exercise

a. Start with having half the group do one rhythm and the other half doing another. The rhythms should blend together and be simple. Then have the group go off on their own rhythm within a similar tempo to serve the purpose of teaching the group about overlapping sounds.

5. Free style over an audio recording 


\section{Closing}
a. Relaxation exercise
b. Check out

\section{Lesson 4}

Objective:

1. Check in

a. Stretch with music audio recording

2. Recap of Afro Cuban Rumba

3. Recap of Gahu

4. Tag you are it, game

i. A participant is chosen to be "it" and they tag other individuals by either pointing at them or saying their name (Faulkner, 2017). While this is happening the rest of the group is doing a foundational rhythm (Faulkner, 2017). To "resist" the tag, the individuals could play three bass notes while keeping in rhythm, but if they lose the rhythm, the tag will stick (Faulkner, 2017).

5. Improv over audio recording

6. Closing
a. Relaxation
b. Stretch
c. Check out

\section{Results}

Warwick Edinburgh Mental Well-being Scale Survey

Each of the fourteen item responses in the Warwick Edinburgh Mental Well-being Scale is scored between 1 (none of the time) to 5 (all the time) (Steward Brown \& Janmohamed, 2008). The total score is calculated by adding the individual item scores (Steward Brown \& Janmohamed, 2008). The minimum score is 14, while the maximum is 70 (Steward Brown \& Janmohamed, 2008). Six eligible participants, eighteen years or older, filled out the pre-test WEMWBS. Four of the respondents were in a moderate level of well-being and two 
demonstrated a high level of well-being. The average score was 53.92. The post-test was given to five individuals with three participants having a moderate level of well-being and two having a high level of well-being. The average score for the post-test was 56.8 ; therefore, there was a 2.88 increase within points.

This increase is also validated through the result percentages of each number present within the pre-test and the post-test. $61.8 \%$ of respondents input a four or higher within the pre-test, while $77.14 \%$ of the respondents input a four or higher post-test after the four-week intervention.

\begin{tabular}{|c|c|c|} 
Scale Number & $\begin{array}{c}\text { Percentages } \\
\text { appeared in } \\
\text { Pre-Test }\end{array}$ & Percentages appeared in Post-test \\
\hline 1 & $0 \%$ & $0 \%$ \\
2 & $7.06 \%$ & $7.14 \%$ \\
\hline 3 & $31.76 \%$ & $15.71 \%$ \\
4 & $31.76 \%$ & $41.43 \%$ \\
5 & $29.42 \%$ & $35.71 \%$ \\
\hline
\end{tabular}

Figure 1. Percentages of scale numbers in the pre and post test

\section{Focus Group}

A constant comparison analysis was used within a three-stage process where the interpretations of the data were compared with the existing data that finalizes with a theme that expresses the overall content (Onwuegbuzie et al., 2009). A total of fourteen codes were uncovered. These codes were shaped into a total of five categories that explored the curriculum, group activities and drumming. The categories were: 1) Community groups should be inclusionary, respectful, and support socialization; 2) Rhythm plays a key role in our lives everyday which makes drum circle more comfortable; 3) The curriculum should cover basic techniques and follow a natural progression; 4) Learning new skills help foster confidence building in participants; and 5) Fostering a strong sense of community is essential for a facilitator. 
The finalized pattern demonstrated:

1. The core of the drum circle is driven by the facilitator fostering a strong sense of community that supports being inclusionary, respectful, and social.

2. Learning new skills, like drumming, in a community group benefits participants confidence when:

a. The curriculum progresses naturally and easily, and because

b. Rhythm is innate and a natural part of everyday life, making drum circles a natural capsule of creativity.

\section{Conclusion}

Participant feedback highlighted the benefits of drum circles in a community setting on wellbeing and the potential for exploration in the specific curriculum for facilitators. All participants' surveys indicated they enjoyed the drum circle and, if the opportunity arose, would be more likely to partake in other creative arts community interventions. Specific words taken from the focus group included peaceful, confident, relaxed, fun, and accomplished. These words are associated with well-rounded well-being. The curriculum fostered a safe space to explore the musical environment without embarrassment or judgement from others. Having a specific curriculum was beneficial to understanding the natural progression of facilitating a drum circle while making the activities participant-centered, where their opinions, likes, and voices were heard while deciding the flow of the class.

\section{Acknowledgements}

This research was carried out in conjunction with the Arts in Medicine Master's Capstone. It was supported by the University of Florida University Scholars program.I thank the UF Arts in Medicine staff and students in their help with assisting this project, as well as all the individuals who participated.

\section{References}

Argyle, E., \& Bolton, G. (2005). Art in the community for potentially vulnerable mental health groups. Health Education (Bradford, West Yorkshire, England), 105(5), 340-354. doi:10.1108/09654280510617178 
Armstrong, K. (2017). The ecology of gahu: Participatory music and health benefits of ewe performance in a canadian drum and dance ensemble. Legon Journal of the Humanities, 27(2), 14. doi:10.4314/ljh.v27i2.2

Ascenso, S., Perkins, R., Atkins, L., Fancourt, D., \& Williamon, A. (2018). Promoting well-being through group drumming with mental health service users and their carers. International Journal of Qualitative Studies on Health and Well-Being, 13(1), 1484219-15. doi:10.1080/17482631.2018.1484219

Azar, N. R. (2020). Rates and patterns of playing-related musculoskeletal disorders in drummers. Medical Problems of Performing Artists, 35(3), 153-161. doi:10.21091/mppa.2020.3020

Brodeur, A., Clark, A. E., Fleche, S., \& Powdthavee, N. (2021). COVID-19, lockdowns and well-being: Evidence from google trends. Journal of Public Economics, 193, 104346. doi:10.1016/j.jpubeco.2020.104346

Carolan, K. (2015). Find Your Beat: Therapeutic Drumming for Parkinson's Disease. Clinical Social Work Journal, 44(2), 179-185. doi:10.1007/s10615-015-0552-3

Clift, S. (2012). Creative arts as a public health resource: Moving from practice-based research to evidence-based practice. Perspectives in Public Health, 132(3), 120-127. doi:10.1177/1757913912442269

Conceptual framework . (2019). Retrieved from https://warwick.ac.uk/fac/sci/med/research/platform/wemwbs/research/framework

Coronavirus disease 2019 (COVID-19). (2020). Retrieved from https://www-cdcgov.lp.hscl.ufl.edu/coronavirus/2019-ncov/community/large-events/considerations-for-eventsgatherings.html

Coughlan, M., \& Cronin, P. (2017). Doing A literature review in nursing, health, and social care (2nd ed.). Thousand Oaks, California: Sage.

Creswell, J., \& Creswell, D. (2018). Research design: Qualitative, quantitative and mixed methods approaches (5th ed.). Thousand Oaks, California: Sage Publications.

Daniel, Y. P. (1991). Changing values in cuban rumba, A lower class black dance appropriated by the cuban revolution. Dance Research Journal, 23(2), 1-10. doi:10.2307/1478752

Davies, C., Knuiman, M., \& Rosenberg, M. (2016). The art of being mentally healthy: A study to quantify the relationship between recreational arts engagement and mental well-being in the general population. BMC Public Health, 16(1), 15. doi:10.1186/s12889-015-2672-7

Dingle, G. A., Clift, S., Finn, S., Gilbert, R., Groarke, J. M., Irons, J. Y., . . Williams, E. J. (2019). An agenda for best practice research on group singing, health, and well-being. Music \& Science, 2, 205920431986171-15. doi:10.1177/2059204319861719

Fancourt, D. (2017). Arts in health designing and researching interventions. United Kingdom: Oxford University Press.

Faulkner, S. (2017). Rhythm to recovery. United Kingdom: Jessica Kinsley Publishers 
Fisher, P.Wellbeing and empowerment the importance of recognition. England, United Kingdom: School of Human and Health Sciences.

Gill, P., Stewart, K., Treasure, E., \& Chadwick, B. (2008). Methods of data collection in qualitative research: Interviews and focus groups. British Dental Journal, doi:10.1038/bdj.2008.192

Hammad, J., El-Guenuni, A., Bouzir, I., \& El-Guenuni, F. (2020).The hand of hope. 20201218 University of Michigan Library. doi:10.3998/jmmh.10381607.0014.202

Hull, A. (2006). Drum circle facilitation . Santa Cruz, California: Village Music Circles.

Hunter, Linda M., et al. “Aboriginal Healing: Regaining Balance and Culture.” Journal of Transcultural Nursing, vol. 17, no. 1, Jan. 2006, pp. 13-22, doi: $10.1177 / 1043659605278937$.

Langevin, H. (2019). The importance of research on health and well-being. Retrieved from https://wwwnccih-nih-gov.lp.hscl.ufl.edu/about/offices/od/director/past-messages/the-importance-of-researchon-health-and-well-being

Little Bit About Depot Park. (2020). Retrieved from http://www.depotpark.org/information.

Mak, H. W., Bu, F., \& Fancourt, D. (2021). Mental health and wellbeing amongst people with informal caring responsibilities across different time points during the COVID-19 pandemic: A population-based propensity score matching analysis Cold Spring Harbor Laboratory. doi:10.1101/2021.01.21.21250045

Mungas, R., \& Silverman, M. J. (2014). Immediate effects of group-based wellness drumming on affective states in university students. The Arts in Psychotherapy, 41(3), 287-292. doi:10.1016/j.aip.2014.04.008

Nagy, E., Allen, C., Hershaw, C., \& Masterton, C. (2021). Title: The effects of a single session musical engagement on changes in mood

National Organization for Arts in Health. (2017). Arts, health, and well-being in America. San Diego, CA: Author.

Onwuegbuzie, A. J., Dickinson, W. B., Leech, N. L., \& Zoran, A. G. (2009). A qualitative framework for collecting and analyzing data in focus group research. International Journal of Qualitative Methods, 8(3), 1-21. doi:10.1177/160940690900800301

Pantelyat, A., Syres, C., Reichwein, S., \& Willis, A. (2016). DRUM-PD: The use of a drum circle to improve the symptoms and signs of parkinson's disease (PD). Movement Disorders Clinical Practice, 3(3), 243-249. doi:10.1002/mdc3.12269

Perkins, R., Ascenso, S., Atkins, L., Fancourt, D., \& Williamon, A. (2016). Making music for mental health: How group drumming mediates recovery. Psychology of Well-Being, 6(1), 1-17. doi:10.1186/s13612-016-0048-0

Perkins, R., Mason-Bertrand, A., Fancourt, D., Baxter, L., \& Williamon, A. (2020). How participatory music engagement supports mental wellbeing Retrieved from https://journals.sagepub.com/doi/pdf/10.1177/1049732320944142 
Plastow, N. A., Joubert, L., Chotoo, Y., Nowers, A., Greeff, M., Strydom, T., . . van Niekerk, E. (2018). The immediate effect of african drumming on the mental well-being of adults with mood disorders: An uncontrolled Pretest-Posttest pilot study. The American Journal of Occupational Therapy, 72(5), 7205345010p1-6. doi:10.5014/ajot.2018.021055

Ranieri, V., Sem Stoltenberg, A., Pizzo, E., Montaldo, C., Bizzi, E., Edwards, S., \& Kamboj, S. (2021). COVID-19 welbeing study: A protocol examining perceived coercion and psychological well-being during the COVID-19 pandemic by means of an online survey, asynchronous virtual focus groups and individual interviews BMJ. doi:10.1136/bmjopen-2020-043418

Schoonenboom, J., \& Johnson, R. B. (2017). How to construct a mixed methods research design. Kölner Zeitschrift Für Soziologie Und Sozialpsychologie, 69(S2), 107-131. doi:10.1007/s11577-0170454-1

Shaw, S. (2020). Hopelessness, helplessness, and resilience: The importance of safeguarding our trainees' mental wellbeing during the COVID-19 pandemic Elsevier. doi:10.1016/j.nepr.2020.102780

Sommer, E. Sonke, J. (2017) Coding Narrative Data. [Powerpoint slides]. Retrieved from ufl.instructure.com

Staff Report. (2020). Alachua county updates COVID-19 rules. Retrieved from https://www.gainesville.com/news/20200517/alachua-county-updates-covid-19-rules

Stewart-Brown, S., \& Janmohamed, K. (2008). Warwick-Edinburgh Mental Well-being Scale (WEMWBS) [Measurement instrument \& User guide]. Retrieved from http://www.ocagingservicescollaborative.org/wp-content/uploads/2014/07/WEMWBSUser-Guide-Version-1-June-2008.pdfe

Thompson, W. F. (2015). Music, thought, and feeling. New York, New York: Oxford University Press.

Traditional drumming \& dance from ghana: Gahu. (2019). Retrieved from https://bellafricana.com/gahutraditional-african-drumming-dance-from-

ghana/\#: :text=According\%20to\%20Ewe\%20Master\%20Drummer\%20Emmanuel\%20Agbeli\%2 0of,than\%20double\%20its\%20original\%20value.\%20Traditional\%20African\%20Instruments 